\title{
Éthique et innovation thérapeutique
}

Vincent Laugel, Ariane Herson, Claire-Cécile Michon, Jean-François Malaterre, Sandrine Segovia-Kueny

$>$ Disposer de médicaments innovants pour soigner les maladies neuromusculaires marque incontestablement un tournant historique. Ce bond en avant scientifique suscite bien des interrogations éthiques, qu'elles concernent la prise en charge des patients, la participation aux essais cliniques ou encore le dépistage néonatal. Autant de pistes de réflexion ouvertes, en trois questions. <

\section{Quel est l'impact des innovations thérapeutiques récentes sur la prise en charge des maladies neuromusculaires?}

\section{Vincent Laugel}

Coordonnateur pédiatrique du Centre de Référence des maladies neuromusculaires, Strasbourg, France.

Vincent.LAUGEL@chru-strasbourg.fr

En pédiatrie, les cardiologues et les oncologues qualifient avec humour la neurologie de discipline «contemplative», car elle dispose de peu de traitements curatifs. L'arrivée de premières thérapies innovantes pose la question d'un éventuel changement de paradigme, avec l'entrée dans une nouvelle ère où la prise en charge symptomatique s'effacerait au profit d'une prise en charge curative. Les consultations des Centres de Référence, et plus largement les hôpitaux, sont-ils adaptés à cette évolution? La façon de parler aux patients et aux familles doit-elle changer? Faudra-t-il «rentabiliser» la prise en charge et pour ce faire privilégier les traitements curatifs au détriment du reste?

Les histoires de deux enfants permettent d'aller plus avant dans la réflexion. Le premier est un petit garçon atteint d'une dystrophie musculaire de Duchenne. Sous corticothérapie, il bénéficie d'un suivi pluridisciplinaire classique. Un traitement par ataluren (Translarna ${ }^{\circledR}$ ) lui est prescrit, sans problème de tolérance particulier. Cet enfant perd ensuite la marche à l'âge de 13 ans. A priori, la thérapie innovante n'a pas eu un impact majeur sur la maladie, même s'il est impossible d'évaluer avec précision son efficacité chez cet enfant. Néanmoins, l'ataluren a un peu modifié la prise en charge, puisque l'enfant a été vu plus souvent en consultation. Il a bénéficié d'un suivi renforcé. La thérapie innovante commence

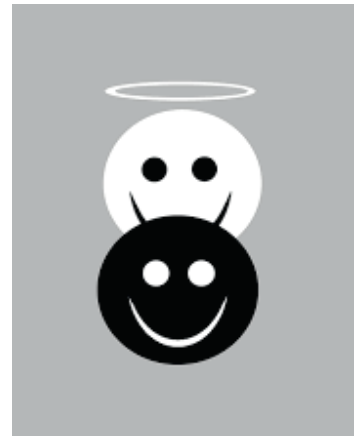

donc peut-être à induire une petite différence, alors même qu'il ne s'agit pas d'un disease modifier: elle ne modifie pas fondamentalement l'histoire de la maladie. Entrer dans un essai clinique, ou dans un traitement innovant, change tout de même l'implication de la famille et le suivi, ce qui impacte la prise en charge symptomatique. C'est un cercle vertueux. Comme le précise la Haute Autorité de Santé (HAS), pour l'instant, le Tanslarna ${ }^{\circledR}$ s'insère sans le modifier dans le schéma thérapeutique actuel de la dystrophie musculaire de Duchenne [1]. La deuxième histoire est celle d'un bébé de 2 mois atteint d'une amyotrophie spinale infantile (SMA) de type 1 , porteur de deux copies du gène SMN2. Ses parents viennent en consultation dans un Centre de Référence parce qu'ils veulent absolument que leur enfant bénéfice du nusinersen (Spinraza ${ }^{\circledR}$ ). Ce traitement est débuté à l'âge de l'âge de 3 mois, à l'issue d'une décision collégiale. L'enfant n'a alors pas d'atteinte respiratoire. Des troubles de la déglutition apparaissent à l'âge de 3,5 mois, puis une insuffisance respiratoire à 4 mois. L'enfant est mis sous ventilation non invasive (VNI) et hospitalisé en réanimation. Dans ce cas, le traitement innovant a eu un impact sur la prise en charge. L'enfant a bénéficié d'une VNI qu'il n'aurait peut-être pas eu s'il n'avait pas été traité par nusinersen. Il a été pris en charge en réanimation au lieu d'être hospitalisé en service conventionnel. L'évolution vers une insuffisance respiratoire terminale a conduit à la décision d'un arrêt du Spinraza ${ }^{\circledR}$. L'enfant est décédé à 5 mois, dans un consensus préservé entre 


\section{L'impact sur la prise en charge, en synthèse}

- Un début de changement de paradigme, mais encore marginal.

- Une nécessité d'intégrer les thérapies innovantes dans la discussion éthique et la prise en charge, avec la famille.

- La science est première et pose les questions éthiques, plus que la maladie.

- Un précédent en oncohématologie pédiatrique, avec l'intrication recherche/soins et curatif/palliatif.

tous les intervenants. Ces deux exemples illustrent à mon sens un changement réel de paradigme, même s'il reste marginal. Les traitements innovants doivent désormais être intégrés très tôt dans la discussion avec les familles, dès le diagnostic.

La science est première et bouscule l'éthique. Elle pose les questions éthiques, plus que la maladie. Et ce qui est éthique à un moment donné peut ne plus l'être dix ans plus tard. Envoyer des volontaires sur la planète Mars n'était pas éthique en 1950 car leur retour sur Terre n'était alors pas garanti. Aujourd'hui, c'est devenu éthique. Ce n'est pas notre conception de l'espace ou l'éthique des scientifiques de la Nasa qui a changé, mais la qualité de la fusée. La science modifie les frontières éthiques. Cela ne signifie pas qu'il faille se décharger de toute réflexion. Il faut réfléchir, mais sans rester bloqué sur des conceptions passées et en connexion étroite avec la science. L'évolution actuelle des maladies neuromusculaires pourrait être comparée à celle intervenue voici plusieurs décennies en oncohématologie pédiatrique, qui est passée de $90 \%$ de décès dans les leucémies à $90 \%$ de traitement. Au-delà d'un résultat en termes de survie, ce changement recouvre une révolution dans les pratiques des équipes qui ont intriqué recherche et soins, curatif et palliatif, la chimiothérapie étant réalisée en même temps que les traitements symptomatiques.

Dans les maladies neuromusculaires, il n'est pas encore naturel de disposer de traitements curatifs. La frontière (un peu dogmatique) entre curatif et symptomatique tend pourtant à s'effacer actuellement. II s'agit avant tout de trouver le bon traitement, pour la bonne personne, au bon moment. Mais est-il possible de tout faire en même temps? Dans un environnement contraint, et un quotidien déjà difficile, l'arrivée des thérapies innovantes apporte de la lumière, mais également une surcharge de travail, à assumer en parallèle des soins courants. Les centres experts n'ont pas forcément été dimensionnés pour la supporter. Les thérapies innovantes changent l'histoire naturelle de la maladie. Comment accompagner les patients dans cette nouvelle histoire naturelle, que nous ne connaissons pas? Les traitements innovants leur ouvrent une perspective, qu'ils sont libres d'emprunter, ou pas. $\diamond$

What is the impact of recent therapeutic innovations on the management of neuromuscular diseases?

\section{Participation à un essai clinique : quels enjeux psychiques pour les patients et leur famille?}

\section{Ariane Herson ${ }^{1}$, Claire-Cécile Michon ${ }^{2}$}

${ }^{1}$ Centre de Référence des maladies neuromusculaires

Pitié-Salpêtrière et Trousseau, Paris, France.

${ }^{2}$ AFM-Téléthon, Évry, France.

ariane.herson@aphp.fr

ccmichon@afm-telethon.fr

Participer à un essai clinique n'a rien d'anodin pour un patient ni pour sa famille. Il n'est pas non plus anodin pour un médecin de proposer un essai clinique. Le malade a ou se construit des représentations autour de l'essai clinique. II a également des représentations de sa maladie et de ses différentes évolutions potentielles dans un contexte où existent des essais cliniques. Dès lors vient inévitablement la question de la guérison, imaginée ou fantasmée comme quelque chose de possible, voire attendue. II devient possible de penser et de parler de guérison. Les professionnels de santé ont également des représentations de tel essai, de telle molécule testée ou de telle technique d'administration. En fonction de la façon dont le médecin parle d'un essai ou d'une molécule, les patients ou les familles ne répondront pas de la même manière à la question : souhaitez-vous participer ou pas? Les personnes concernées et les professionnels ont enfin des représentations, mais aussi des fantasmes, autour de nouvelles technologies comme la thérapie génique. Que fait-on chez l'humain quand on touche à ses gènes?

L'arrivée des essais cliniques dans les maladies neuromusculaires modifie la relation patient - médecin. Le patient a de nouvelles attentes. II peut choisir le médecin en capacité de lui proposer un essai. Le médecin n'annonce pas le diagnostic d'une maladie neuromusculaire de la même façon lorsqu'il n'a rien d'autre à proposer que la rééducation et les aides au confort habituel, et lorsqu'il peut de surcroît proposer la participation à un essai. Le tout premier enjeu est celui de la décision. Est-ce que je participe ou pas à cet essai clinique? Beaucoup de facteurs, rationnels et irrationnels, entrent en jeu dans cette décision, qui peut être un moment accompagnée de façon pluridisciplinaire par le médecin, l'attaché de recherche clinique (Arc), le psychologue et l'AFM-Téléthon. Tous ces intervenants peuvent aider, chacun à leur façon, mais l'autonomie de la décision demeure essentielle. Mais ai-je le droit de refuser? Ai-je vraiment le choix puisque je n'ai 


\section{La déclaration d'Helsinki le dit}

« Lorsqu'une personne considérée comme incapable de donner un consentement éclairé est en mesure de donner son assentiment concernant sa participation à la recherche, le médecin doit solliciter cet assentiment en complément du consentement de son représentant légal ».

\section{Les enjeux psychiques, en synthèse}

-Ai-je le droit de refuser? Ai-je le choix?

- Comment l'enfant peut s'approprier la décision et

s'impliquer tout au long de l'essai?

- L'accompagnement relationnel est devenu une exigence éthique. finalement pas d'alternative? Certaines personnes décident de ne pas participer. Comme l'a souligné une maman, «participer à un essai clinique, c'est une transformation du quotidien », qui devient rythmé par les rendez-vous et les autres contraintes liés à la participation à l'essai. En aval, la sortie de l'essai clinique pour un évènement indésirable ou la fin du protocole est souvent associée à un sentiment d'abandon, en raison de la fin du lien particulier, un peu privilégié, qui s'est tissé avec l'équipe. De façon paradoxale, ce sentiment d'abandon apparait parfois en cas de passage de l'essai au traitement, une situation $a$ priori très favorable et positive mais qui se traduit par la fin d'une période où le patient s'est senti très soutenu et très suivi.

$\varepsilon n$ pédiatrie, les essais cliniques présentent des spécificités, à commencer par la place particulière de l'enfant et de son savoir sur la maladie. Lors des premiers essais pédiatriques, nous avons pu constater que souvent l'annonce de la maladie n'avait pas été réellement faite à l'enfant, ou alors de façon partielle. A un moment donné, il y a nécessité de faire cette annonce, de lui parler de sa maladie c'est-à-dire de la nommer, de lui expliquer pourquoi il chute et pourquoi il court moins vite que les autres à l'école. Le moment où commencent à apparaître des essais cliniques est parfois aussi l'occasion de parler de la maladie en famille. Une autre spécificité en pédiatrie porte sur la responsabilité parentale. Ce sont les parents qui décident de la participation à un essai de leur enfant. Dans cette situation, il s'agit davantage de chercher l'assentiment de l'enfant que son consentement pour participer, comme le précise la déclaration d'Helsinki de la World medical association révisée en 2013 à propos des personnes vulnérables [2].

La décision de participation confronte les parents à des dilemmes, des conflits internes autour de ce qu'ils peuvent décider pour le bien de leur enfant, avec les risques éventuels que l'essai peut comporter. Une difficulté particulière survient lorsque le protocole prévoit un groupe traité par placebo, défini par tirage au sort. Si une telle méthodologie est indispensable à titre collectif, l'accepter à titre individuel signifie preuve d'altruisme, et donc d'altruisme par procuration pour les parents. La question essentielle est: comment l'enfant peut-il s'approprier la décision et s'impliquer tout au long de l'essai ? Par expérience, il peut remettre en question son assentiment initial à un moment ultérieur au cours de l'essai clinique, voire au cours du traitement. Cette remise en question participe d'un processus décisionnel qu'il n'a pas forcément pu mettre en œuvre au tout premier temps.

Chez l'enfant comme chez l'adulte, l'avènement des essais cliniques représente un évènement ambivalent, à la fois très positif parce que susceptible d'avoir une influence favorable sur l'évolution de la personne, mais aussi très perturbant sur le plan psychique. Une personne peut se voir améliorer par exemple sur le plan moteur et, de façon très paradoxale, exprimer toutes les modifications, la perturbation, les bouleversements que cela représente pour elle sur le plan psychique. C'est pourquoi elle doit être accompagnée. Comme le dit Christian Réveillère, psychologue conseil à l'AFM-Téléthon, « l'accompagnement relationnel est devenu une exigence éthique ». Les psychologues des Centres de Référence des maladies neuromusculaires ont à cœur de s'impliquer dans cet accompagnement. Les médecins disent manquer de temps. Certaines informations nécessitent d'être dites en plusieurs fois, de différentes façons et à différents moments. C'est ce que permettent les différents interlocuteurs. Les psychologues des consultations pluridisciplinaires sont prêts à s'investir dans cette mission. Ils sont là pour aider à anticiper l'éventualité d'une déception (presque inévitable) et à gérer l'après-coup et ses effets. $\diamond$

Joining a clinical trial: which emotional impact for patients and their families?

\section{Faut-il étendre \\ le dépistage néonatal aux maladies \\ neuromusculaires et selon quelles modalités?}

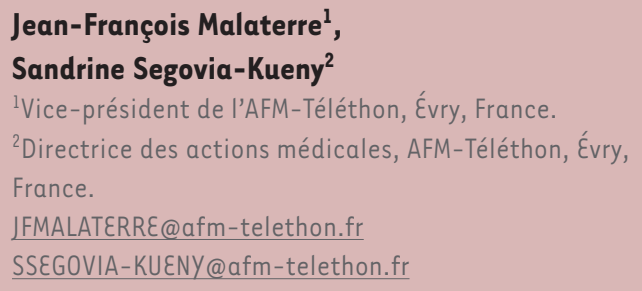

Le dépistage dit « individuel » est réalisé à la suite d'un examen clinique ou biologique dont les résultats sont évocateurs d'une maladie. Le dépistage « en cascade » est proposé aux membres d'une famille où une personne a été diagnostiquée comme atteinte d'une maladie, par exemple la dystrophie musculaire de Duchenne ou la maladie de Steinert. Le troisième type de dépistage est réalisé à l'échelle d'une population [3]. II répond à un 


\section{Les dix critères de Wilson et Jungner}

1. La maladie constitue un problème important de santé, en raison de sa fréquence.

2. Un traitement d'efficacité démontrée peut être administré aux personnes chez lesquelles la maladie est décelée.

3. Les moyens appropriés de diagnostic et de traitement sont disponibles.

4. La maladie est décelable pendant la phase de latence (pré-symptomatique) ou au début de la phase symptomatique.

5. Une épreuve ou un examen de dépistage efficace existe.

6. Ce test est acceptable pour la population.

7. L'histoire naturelle de la maladie est connue, notamment son évolution de la phase pré-symptomatique à la phase symptomatique.

8. Le choix des personnes qui recevront un traitement est opéré selon des critères préétablis.

9. Le coût de la recherche des personnes atteintes n'est pas disproportionné par rapport au coût global des soins médicaux.

10. La recherche des personnes atteintes est continue et n'est pas considérée comme une opération réalisée « une fois pour toutes ».

certain nombre de critères, définis dès 1968 par Wilson et Jungner et entérinés par l'Organisation mondiale de la santé (OMS) [4].

La France a été précurseur en ce domaine avec le déploiement, dès 1972, d'un premier programme de dépistage néonatal ${ }^{1}$. Le dernier en date à avoir été généralisé concerne la mucoviscidose, adopté alors même qu'il ne remplit pas strictement les critères de Wilson et Jungner puisqu'aucun traitement curatif n'est disponible pour cette maladie. Un traitement symptomatique précoce a toutefois une influence positive sur la qualité de vie et sur la durée de vie des enfants atteints de mucoviscidose.

Quelle que soit la maladie recherchée, un dépistage néonatal bénéficie à l'enfant, à ses parents et à la société. Pour l'enfant, il signifie d'abord la suppression de l'errance diagnostique. Un deuxième bénéfice est d'avoir accès de façon précoce aux traitements, mais aussi à la prise en charge sociale. Les parents bénéficient également de la suppression de l'errance diagnostique et accèdent plus tôt au conseil génétique. Ils peuvent anticiper des choix importants pour leur qualité de vie. La société enfin peut, grâce au dépistage néonatal, éviter des cas et réduire les coûts de santé.

La suppression de l'errance diagnostique constitue un avantage primordial pour les maladies neuromusculaires [5], où l'incertitude peut durer actuellement plusieurs années. Ainsi, le diagnostic de myopathie de Duchenne n'a été posé chez certains enfants qu'à l'âge de huit ans, alors même que les premiers signes de la maladie apparaissent vers l'âge de trois ans. Le dépistage néonatal permettrait également de compléter la connaissance de l'histoire naturelle des pathologies neuromusculaires, dès le plus jeune âge. II permettrait enfin, et sur-

${ }^{1}$ En France a été instauré un dépistage systématique, chez tous les nouveau-nés (dépistage néonatal) de la phénylcétonurie en 1972, puis de l'hypothyroïdie congénitale (1978), de l'hyperplasie congénitale des surrénales (1995), de la drépanocytose (1989 en Outre-Mer et 1995 en métropole) et la mucoviscidose (2002). tout, à toutes les personnes concernées d'accéder aux nouvelles approches thérapeutiques. Nous arrivons à un moment de l'histoire de la médecine où s'annoncent des thérapies qui font envisager de pouvoir enfin éradiquer les maladies neuromusculaires. Cet espoir qui est le nôtre depuis bien longtemps (I'AFM-Téléthon fête cette année ses 60 ans) commence à prendre corps. Nous pouvons aujourd'hui espérer rentrer dans l'ère des traitements. Ils ne sont pas encore curatifs. Cependant, un certain nombre de médicaments actuellement en essais cliniques ou en passe d'arriver sur le marché sont porteurs d'espoirs très concrets, notamment dans l'amyotrophie spinale infantile et la dystrophie musculaire de Duchenne (DMD). Pour cette dernière, un essai clinique basé sur le saut d'exon arrive en Europe. II inclura de jeunes enfants atteints d'une DMD âgés de 6 mois à 4 ans. La France, qui aime bien les challenges, a choisi des critères d'inclusion encore plus restrictifs, pour cibler les enfants DMD âgés de 6 mois à 2 ans seulement. En l'absence de dépistage néonatal de la maladie, ils ne sont pas légion à être diagnostiqués dans cette tranche d'âge. Dans l'enquête sur la myopathie de Duchenne réalisée par l'AFM-Téléthon, l'âge moyen du diagnostic était de 6 ans [6]. D'autres publications l'évaluent plutôt à 5 ans. Sachant qu'une centaine de nouveau-nés atteints de la DMD voie le jour chaque année en France, notre pays compte environ 500 à 600 enfants de 0 à $5-6$ ans à ne pas se savoir atteints. L'essai de saut d'exon va concerner à peu près $10 \%$ des enfants Duchenne, ce qui signifie que 50 enfants qui pourraient en bénéficier ne seront pas inclus car ils ne sont pas encore diagnostiqués.

Pour autant, faut-il dépister les maladies neuromusculaires à la naissance? Chacune des familles concernées a sa propre réponse, forgée par son histoire. Pour certains parents, une information précoce a permis d'anticiper l'avenir et de mettre en place rapidement des soins pour leur enfant. Pour d'autres, «ne pas savoir» trop tôt leur a permis de profiter d'un temps de répit (ou d'innocence) durant lequel ils ont pu développer avec leur enfant des interactions qui auraient été différentes s'ils l'avaient su malade.

À l'échelle non plus individuelle mais collective, nous devons dès à présent réfléchir à l'instauration d'un dépistage néonatal national, sans attendre de disposer de traitements curatifs. Quelles seraient les maladies éligibles? Comment les dépister? Selon quelles modalités? Une réflexion est en cours dans le Groupe d'intérêt Duchenne/Becker de l'AFM-Téléthon. Le sujet a été très débattu, mais le groupe d'intérêt s'est prononcé pour le dépistage néonatal de la DMD. A partir du moment où le diagnostic est posé à un stade précoce, 
Le dépistage néonatal dans les maladies neuromusculaires, en synthèse

- Quels bénéficiaires?

L'enfant

Les parents

La société

- Mise en place d'un groupe de réflexion

Quelles maladies?

Quel accompagnement?

Quels enjeux pour la famille et pour la société?

et a fortiori à la naissance, comment accompagner les parents dans la gestion de cette situation? L'expérience de dépistage néonatal de la DMD menée dans la région lyonnaise durant dix ans atteste de la nécessité de les accompagner [7]. Des recommandations dédiées devraient être élaborées. $\nabla$

Should newborn screening be extended to neuromuscular diseases and how?

\section{LIENS D'INTÉRÊT}

Les auteurs déclarent n'avoir aucun lien d'intérêt concernant les données publiées dans cet article.

\section{RÉFÉRENCES}

1. Haute Autorité de Santé. Commission de la transparence. Avis du 5 juillet 2017 - Ataluren (sur www.has-sante.fr).

2. Déclaration d'Helsinki de la World medical association - Principes éthiques applicables à la recherche médicale impliquant des êtres humains, amendés par la $64^{e}$ Assemblée générale de la WMA, Fortaleza (Brésil), octobre 2013 (sur www.wma.net)

3. Ross LF, Clarke AJ. A Historical and current review of newborn screening for neuromuscular disorders from around the world: lessons for the United States. Pediatr Neurol $2017 ; 77$ : 12-22.

4. Wilson JMG, Jungner F. Principes et pratiques du dépistage des maladies. Cahiers de santé publique $n^{\circ} 34$. Geneva : Organisation mondiale de la santé, 1970 (sur www.who.int).

5. Malaterre JF. Maladie rare et diagnostic précoce. La dystrophie musculaire de Duchenne-Becker. Accompagnement des personnes atteintes de maladies génétiques et de leur famille. Mémoire de diplôme universitaire. Université Paris VI - Faculté de médecine Pitié-Salpêtrière, année 20022003.

6. AFM-Téléthon. Dystrophies musculaires de Duchenne et Becker : vécu et attentes des personnes concernées. Savoir et comprendre, Enquêtes, octobre 2014 (sur www.myobase.org).

7. Plauchu H, Cordier MP, Carrier HN, et al. Dépistage néonatal systématique de la dystrophie musculaire de Duchenne : bilan après dix ans d'expérience dans la région de Lyon (France). J Genet Hum 1987 ; 35 : 217-30.

Ethics and therapeutic innovation

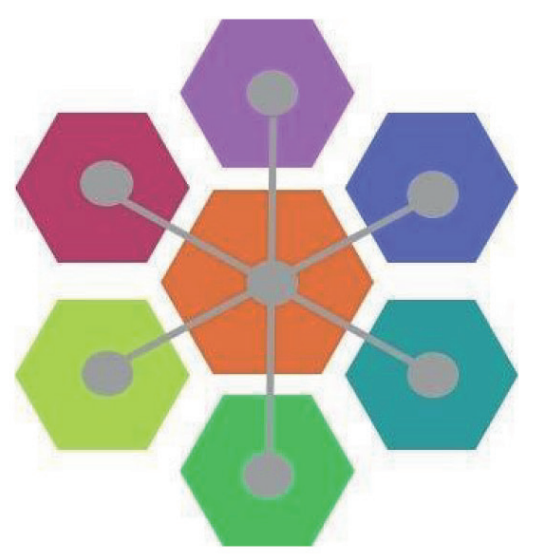

\section{Global Registry for COL6-related dystrophies}

\section{Registre global des dystrophies liées au collagène de type VI}

S'inscrire sur: www.collagenb.org

Ou contactez-nous par e-mail à l'adresse : collagenbregistry@ncl.ac.uk

La traduction française sera bientôt disponible sur le site web.

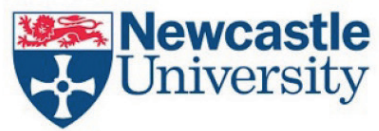

$\int \mathrm{TR}$ TREAT-NMD

\section{Jöhn}

Walton
Muscular Dystrophy UK Fighting muscle-wasting conditions
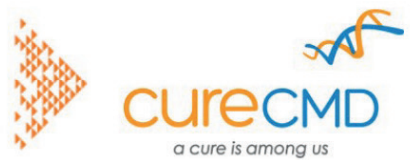

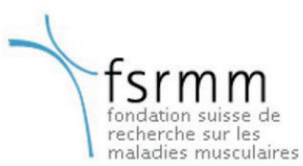

